\title{
Greenness Assessment of Spectrofluorometric Method for Quantification of Ertugliflozin: Application to Dosage Form and Human Urine
}

\author{
Rasha M. Ahmed \\ ${ }^{a}$ Department of Pharmaceutical Chemistry, Faculty of Pharmacy, Misr International University, Cairo, \\ Egypt.
}

Received on: 25. 9. 2021

Revised on: 01. 10. 2021

Accepted on: 06. 10. 2021

*Correspondence Author:

Tel: +201223682995

E-mail address:

rasha_ahmed@miuegypt.edu.eg

\begin{abstract}
The proposed work describes an ecofriendly, cost-effective, and sensitive spectrofluorometric method for the determination of ertugliflozin (ERT) in bulk material, pharmaceutical dosage form, and human urine. The method was developed by measuring the native fluorescence of the cited drug at $\lambda_{\mathrm{em}}=334 \mathrm{~nm}$ after excitation at $\lambda_{\mathrm{ex}}=270$ $\mathrm{nm}$ using water as a solvent. Different variables affecting the native fluorescence nature of ERT were successfully studied to obtain the optimum conditions of the proposed method. The assay was validated according to ICH guidelines for analytical and bioanalytical method validation and satisfactory results were obtained; percentage recoveries ranged between $99.47 \%$ and $101.36 \%$ and between $99.19 \%$ and $101.90 \%$ upon analysis of ERT in dosage form and human urine respectively. No significant matrix effect was observed while measuring ERT in pharmaceutical dosage form or in human urine. The proposed method is considered the first spectrofluorometric method for quantification of ertugliflozin in dosage forms and human urine and can be applied to biological samples obtained from a pharmacokinetic study of ERT. Moreover, the method greenness was investigated by applying two different techniques and a comparative study was performed between the proposed method and other reported methods.
\end{abstract}

Keywords: Ertugliflozin; spectrofluorometry; greenness assessment; human urine.

\section{Introduction}

Ertugliflozin (ERT), has the chemical formula (1S,2S,3S,4R,5S)-5-[4-Chloro-3- (4-ethoxybenzyl) phenyl]-1-(hydroxymethyl)-6,8-dioxabicyclo[3.2.1] octane-2,3,4-triol (Fediuk et al. 2020) (Figure 1). ERT is a sodium-glucose cotransporter 2 (SGLT2) inhibitor that is used as an insulin-independent hyperglycemic agent for the treatment of type II Diabetes Mellitus (DM) (Dawra et al. 2019a). Its action appears to be through glucose reabsorption from glomerular filtrate and also through elevation of urinary excretion of glucose, leading to reduction of glucose in blood circulation 
(Nguyen and White 2019). ERT has been approved by the food and drug administration in 2017 (European Medicines Agency Committee for Medicinal Products for Human Use 2018).

After oral administration of ERT, the peak plasma concentration was observed after two hours with maximum plasma concentration $268 \mathrm{ng} / \mathrm{mL}$ and halflife 16.6 hours followed by a rapid decline due to distribution and elimination (Dawra et al. 2019). ERT is metabolized by glucuronidation (major metabolic pathway) via uridine diphosphate glucuronosyltransferase and oxidation (minor metabolic pathway) via the hepatic enzyme cytochrome P4503A4 (CYP 3A4) (Fediuk et al. 2021). ERT is metabolized into six metabolites and $35.3 \%$ of the administrated dose is excreted unchanged (Cinti et al. 2017).

Since concentrations of unchanged ERT in human plasma and urine are very low, it was necessary to develop a sensitive and rapid analytical method for the quantification of ERT in human urine and human plasma. A spectrofluorimetric technique has become the method of choice for the analysis of drugs in biological fluids because of its sensitivity and simplicity compared to chromatographic techniques (Ahmad et al. 2018; Al-Attas et al. 2017; Zidan et al. 2018). Little information on the determination of ERT is available and no spectrofluorometric method was observed in the literature.

Shafaat et al.(Syed Wajahat S. et al. 2019) reported an LC-UV method for simultaneous determination of ERT and metformin in dosage form by HPLC-UV at $220 \mathrm{~nm}$ with a limit of quantification (LOQ) of 1.5 $\mu \mathrm{g} / \mathrm{mL}$ for ERT. Kumari et al.(Kumari et al. 2020) used the same technique as Shafaat et al. but using a different analytical column at $224 \mathrm{~nm}$ with a linear range of $0.93-5.62 \mu \mathrm{g} / \mathrm{ml}$. However, the LOQ in this reported article was found low $(0.076 \mu \mathrm{g} / \mathrm{ml})$ when compared to $0.93 \mu \mathrm{g} / \mathrm{ml}$ in a linear range of ERT. Rao et al. (P.venkateswara rao et al. 2018) reported an LC-UV method for simultaneous determination of ERT and sitagliptin in pharmaceutical dosage form by HPLC-UV at $240 \mathrm{~nm}$. Reddy B et al. (Anjaneyulu Reddy B et al. 2019) reported a LCcharged aerosol detection technique for simultaneous determination of ERT and sitagliptin in pharmaceutical dosage form with LOQ of 0.24 $\mu \mathrm{g} / \mathrm{mL}$ for ERT. Qiu et al. (Qiu et al. 2019) published an UPLC-MS/MS method for simultaneous quantification of ERT and sitagliptin in rat plasma. Han et al. (Han et al. 2019) reported HPLC- fluorescence for determination of ERT in rat plasma with a linear range of $1-2000 \mathrm{ng} / \mathrm{mL}$.

In this paper, a simple, ecofriendly, sensitive, and cost-effective spectrofluorimetric method will be presented for the determination of ERT in pharmaceutical dosage form and human urine. The developed and validated method can be successfully applied to urine samples from a pharmacokinetic study of ERT delivered via oral administration of ERT dosage forms. In addition, the greenness of the proposed method was evaluated using the green analytical procedure index (GAPI) and Analytical GREEnness metrics (AGREE) approaches.

\section{Experimental}

\subsection{Instrumentation}

Shimadzu spectrofluorometer (model: RF 5301 PC, Japan), with Xenon arc lamp, was used to measure the fluorescence intensities. Excitation and emission Slit widths were set at $5 \mathrm{~nm}$ and $1 \mathrm{~cm}$ quartz cell was used. Jenway pHmeter 3510 (Cole-Parmer, Staffordshire, UK) was used to perform $\mathrm{pH}$ measurements.

\subsection{Chemicals and reagents}

ERT was obtained from Hekma pharmaceutical industries, Cairo, (Cairo, Egypt). ERT $^{\circledR}$ tablets $\mathrm{F}(14 / 231) \mathrm{A}$ were purchased from local pharmacies and each tablet contained $5 \mathrm{mg}$ of ertugliflozin. Acetonitrile, methanol (Sigma-Aldrich, Germany) used throughout the work were of HPLC grade while the rest of the solvents and reagents were of analytical grade. Hydrochloric acid, ethanol, sodium hydroxide, acetone, and ethyl acetate were purchased from El-Nasr Pharmaceutical Chemicals Co. (Egypt).

\subsection{Standard solutions}

Stock solutions of ERT were prepared in water at a concentration of $1000 \mu \mathrm{g} / \mathrm{mL}$. Standard working solutions with a concentration range between 1-40 $\mu \mathrm{g} / \mathrm{mL}$ were prepared by diluting the stock solutions with the same solvent. Both stock and working standard solutions were stored at $4^{\circ} \mathrm{C}$.

\subsection{Construction of the calibration curve}

Different volumes of ERT working solution were 


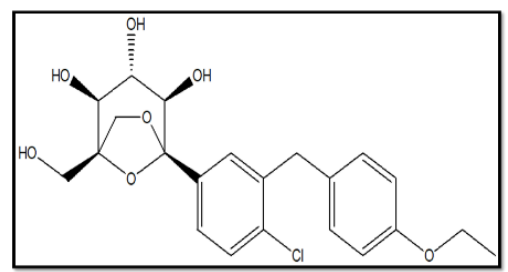

Figure 1: Chemical Structure of ERT.

Table 1: Analytical parameters of the proposed spectrofluorometric method for determination of ERT in aqueous medium.

\begin{tabular}{lll}
\hline Parameter & $\begin{array}{c}\text { Spectrofluorometric } \\
\text { aqueous medium }\end{array}$ & method in \\
\hline$\lambda_{\text {ex }}$ & $270 \mathrm{~nm}$ \\
$\lambda_{\text {em }}$ & $334 \mathrm{~nm}$
\end{tabular}

Linearity:

Regression equation

Range (ng/mL)

Correlation coefficient (r)

Slope

Intercept

S.D of slope

R.S.D. of slope

S.D of intercept

LOD(ng/mL)

LOQ (ng/mL)

Precision:

Repeatability (Intraday)

(RSD)*

QCL (200 ng/mL)

1.53

QCM (500 ng/mL)

0.82

QCH (800 ng/mL)

0.71

Intermediate precision (Inter-day)

(RSD )*

QCL $(200 \mathrm{ng} / \mathrm{mL})$

0.36

QCM (500 ng/mL)

0.22

QCH (800 ng/mL)

0.12

Accuracy:

(Mean \% Recovery \pm SD) $* *$

QCL (200 ng/mL)

$99.47 \pm 1.16$

QCM (500 ng/mL)

$101.36 \pm 0.17$

QCH (800 ng/mL)
$Y_{l}=0.86 x+189.04$

$50-1000$

0.9992

0.86

189.04

1.16

4.5

17.27

52.32

RSD: relative standard deviation, $* *$ Expressed mean of three replicates. 


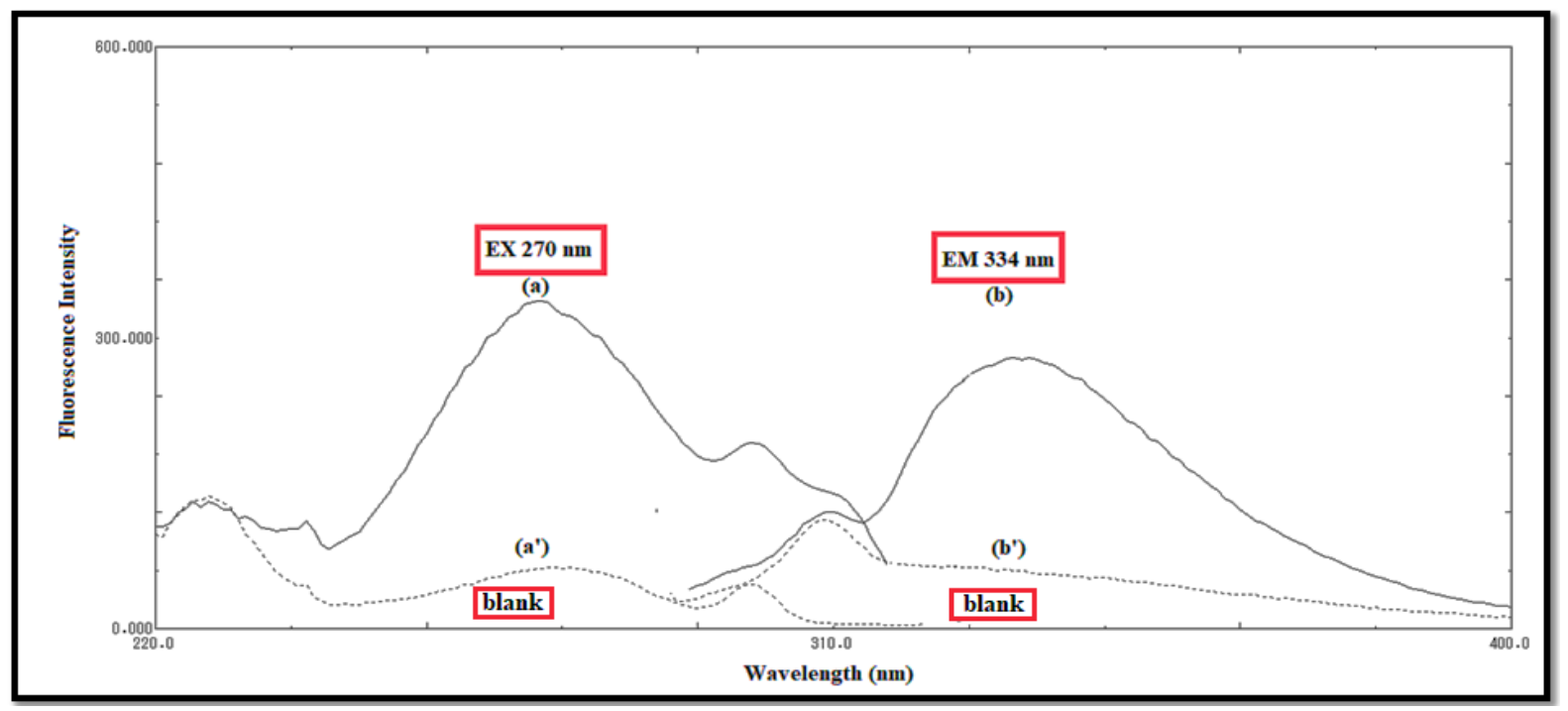

Figure 2: Excitation (a', b') and emission spectra (a,b) of ERT in ethanolic solution.

Table 2: Application of the proposed method for determination of ERT in spiked human urine

\begin{tabular}{ll}
\hline Parameter & $\begin{array}{l}\text { Spectrofluorometric method in } \\
\text { human urine }\end{array}$ \\
\hline$\lambda_{\text {ex }}$ & $270 \mathrm{~nm}$ \\
$\lambda_{\text {em }}$ & $334 \mathrm{~nm}$ \\
Linearity: & $y=1.2453 x+267.52$ \\
Regression equation & $50-1000$ \\
Range $(\mathrm{ng} / \mathrm{mL})$ & 0.9991 \\
Correlation coefficient $(\mathrm{r})$ & 1.23 \\
Slope & 267.52 \\
Intercept & \\
QC samples & $\underline{\text { Accuracy* } \pm \mathrm{RSD} * *}$ \\
Repeatability $(\mathrm{Intraday})$ & $101.44 \pm 1.82$ \\
LLOQ $(50 \mathrm{ng} / \mathrm{mL})$ & $100.39 \pm 2.00$ \\
QCL $(100 \mathrm{ng} / \mathrm{mL})$ & $101.69 \pm 0.58$ \\
QCM $(300 \mathrm{ng} / \mathrm{mL})$ & $99.19 \pm 0.81$ \\
QCH $(500 \mathrm{ng} / \mathrm{mL})$ & $\underline{\text { Accuracy* }} \pm \mathrm{RSD} * *$ \\
Intermediate precision (Inter-day) & $100.97 \pm 1.52$ \\
LLOQ $(50 \mathrm{ng} / \mathrm{mL})$ & $100.58 \pm 1.32$ \\
QCL $(100 \mathrm{ng} / \mathrm{mL})$ & $101.90 \pm 0.96$ \\
QCM $(300 \mathrm{ng} / \mathrm{mL})$ & $99.30 \pm 0.06$ \\
QCH $(500 \mathrm{ng} / \mathrm{mL})$ &
\end{tabular}

*Average of five determinations, **Relative standard deviation. 
transferred into a set of $10-\mathrm{mL}$ volumetric flasks, and all the volumetric flasks were completed to the mark with water to reach final concentrations in the range of $50-1000 \mathrm{ng} / \mathrm{mL}$. The prepared solutions were measured at $334 \mathrm{~nm}$ after excitation at $270 \mathrm{~nm}$. Blank was performed and fluorescence intensities were determined. The calibration curve was constructed by plotting relative fluorescence intensity (RFI) values against corresponding concentrations to construct the calibration curve then the regression equation was computed.

\subsection{Analysis of ERT pharmaceutical preparations}

Ten film-coated tablets (Stiglarto®) each contains $5 \mathrm{mg}$ ertugliflozin, 40 tablets were accurately weighed, grinded, and thoroughly mixed. An accurate portion of the powder equivalent to $100 \mathrm{mg}$ of ERT was cautiously transferred into a $100-\mathrm{mL}$ volumetric flask followed by the addition of $50 \mathrm{~mL}$ of water. The prepared solution was sonicated for 15 min then filtered and diluted with water to the appropriate volume to reach a final concentration of $1000 \mu \mathrm{g} / \mathrm{mL}$. Into a $100 \mathrm{~mL}$-volumetric flask, $1 \mathrm{~mL}$ of the filtrate was transferred and diluted to reach a concentration of $1 \mu \mathrm{g} / \mathrm{mL}$. Different aliquots of the prepared solution were transferred into $10 \mathrm{~mL}$ volumetric flasks followed by dilution to the mark with water. The solutions were measured at $334 \mathrm{~nm}$ after excitation at $270 \mathrm{~nm}$ and relative fluorescence intensities were recorded then the corresponding concentrations were calculated using the computed regression equation.

\subsection{Analysis of ELG in spiked human urine}

Human free drug urine samples $(900 \mu \mathrm{L})$ were spiked with $100 \mu \mathrm{L}$ of ERT working standard solution containing different concentrations of ERT $(5-100 \mu \mathrm{g} / \mathrm{mL})$ in a set of $10 \mathrm{~mL}-$ volumetric flasks separately followed by the addition of distilled water up to the mark to reach concentration range between $50 \mathrm{ng} / \mathrm{mL}-1000 \mathrm{ng} / \mathrm{mL}$. The average fluorescence intensities were measured at $334 \mathrm{~nm}$ after excitation at $270 \mathrm{~nm}$ and in the same way, the blank experiments were carried out then the regression equation was computed.

\section{Results and discussion}

The aim of this work was to perform a fast, ecofriendly, and economic spectrofluorometric method for evaluation of ERT in different matrices (dosage form and human urine) because it was found that there was no spectrofluorometric technique for determination of ERT in bulk, dosage form, human plasma and/or human urine. Different experimental variables that have an impact on the fluorescence behavior of ERT were studied cautiously by one factor at a time approach.

\subsection{Optimization of spectrofluorometric parameters}

ERT exhibits high native fluorescence at $\lambda_{\mathrm{em}} 334 \mathrm{~nm}$ after using excitation wavelength at $270 \mathrm{~nm}$. Excitation and emission spectra of ERT in aqueous medium and urine are presented in Figure 2. Different variables affecting the fluorescence nature of ERT were studied such as: diluting solvent effect and $\mathrm{pH}$ effect.

During optimization of spectrofluorometric parameters, the impact of diluting solvent on the fluorescence behavior of ERT was studied using different solvents as water, ethanol, methanol, acetonitrile, ethyl acetate, acetone, $0.1 \mathrm{M} \mathrm{HCl}$, and $0.1 \mathrm{M} \mathrm{NaOH}$. In the chemical structure of ERT, there are seven oxygen atoms (hydrogen acceptor atoms) that have the liability to form a hydrogen bond between solvents. The strong hydrogen bonding was formed between cited drug and water resulting in high RFI compared to other used solvents as presented in Figure 3. Upon using different diluting solvents, there was no change in the position of excitation or emission wavelengths.

\subsection{Analytical Method validation in aqueous medium}

Assay validation was carried out according to $\mathrm{ICH}$ guidelines for bioanalytical methods validation (Harron 2013). The following validation criteria were investigated: linearity, accuracy, precision, the limit of detection, and the limit of quantitation.

\subsubsection{Linearity and sensitivity}

Linearity was evaluated using six concentration levels covering the range of $50-1000 \mathrm{ng} / \mathrm{mL}$ for ERT. The regression equation was $(\mathrm{Y}=0.8685 \mathrm{X}+$ $\left.189.04, r^{2}=0.9992\right)$ where $Y$ was the RFI of the ERT and X was the concentration of ERT in $\mathrm{ng} / \mathrm{mL}$. The statistical parameters of the proposed method were summarized in Table $\mathbf{1}$ and the observed data reveals acceptable linearity of the proposed method. The sensitivity of the method was evaluated by calculating the limit of detection 
Table 3: Application of the proposed method for determination of ERT in pharmaceutical dosage form and application of standard addition technique

\begin{tabular}{|c|c|c|c|c|}
\hline \multirow{2}{*}{ Dosage form } & \multirow{2}{*}{$\begin{array}{l}\text { Claimed } \\
(\mathrm{ng} / \mathrm{mL})\end{array}$} & \multirow{2}{*}{$\begin{array}{c}\% \text { Found } \\
(\text { Mean } \pm \text { SD })\end{array}$} & \multicolumn{2}{|c|}{ Standard addition technique } \\
\hline & & & $\begin{array}{c}\text { Pure added } \\
(\mathrm{ng} / \mathrm{mL})\end{array}$ & $\begin{array}{c}\text { \% Recovery }^{* * *} \\
(\text { Mean } \pm \text { SD })\end{array}$ \\
\hline $\begin{array}{c}\text { Steglarto }{ }^{\circledR} \text { tablets } \\
\text { (contains } 5 \mathrm{mg} \\
\text { ertugliflozin) }\end{array}$ & 200 & $98.80 \pm 0.59$ & $\begin{array}{l}100 \\
200 \\
300\end{array}$ & $\begin{array}{l}100.25 \pm 1.36 \\
101.74 \pm 0.71 \\
100.85 \pm 0.42\end{array}$ \\
\hline
\end{tabular}

\footnotetext{
**Average of three determinations.
}

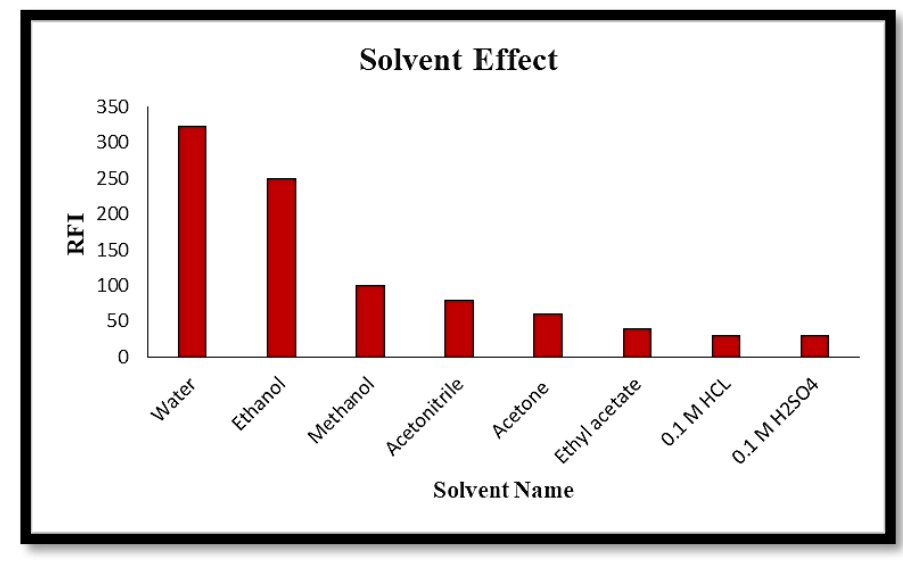

Figure 3: Effect of different solvents on the fluorescence intensity of ERT.

(LOD) and limit of quantitation (LOQ) of the proposed assay using the following equations; LOD $=3.3 \mathrm{~S}_{\mathrm{a}} / \mathrm{b}$ and $\mathrm{LOQ}=10 \mathrm{~S}_{\mathrm{a}} / \mathrm{b}$, where: $\mathrm{S}_{\mathrm{a}}$ is the standard deviation of the intercept while $b$ is the slope of the calibration curve. The LOD and LOQ were calculated and found to be 17.27 and 52.32 $\mathrm{ng} / \mathrm{mL}$ indicating the high sensitivity of the method.

\subsubsection{Accuracy and precision}

The assessment of the accuracy of the developed method was carried out by analysis of ERT at three different concentrations (QCL, QCM, and QCH) using the same analytical procedure. The evaluation of accuracy was performed by calculating the percentage recoveries and corresponding standard deviation. The observed results were found to be close to $100 \%$ revealing good accuracy of the proposed method as illustrated in Table 1.

Analysis of ERT at three concentration levels (QCL, QCM, and QCH) was carried out and the intraday and inter-day precision of the method were evaluated. The obtained results clearly demonstrated the developed assay possessed acceptable precision with adequate relative standard deviation (RSD) as shown in Table 1.

\subsection{Analytical method validation in human urine}

The spectrofluorometric method was applied to the quantification of ERT in spiked human urine. The proposed method was validated according to the 
Table 4: Comparison of the spectrofluorometric method and other reported methods

\begin{tabular}{|c|c|c|c|c|c|c|}
\hline Proposed Method & $\begin{array}{c}\text { Reported } \\
\text { (Syed et al. 2019) }\end{array}$ & $\begin{array}{c}\text { Reported } \\
\text { (Kumari et al. 2020) }\end{array}$ & $\begin{array}{c}\text { Reported } \\
\text { (P venkat. et al. 2018) }\end{array}$ & $\begin{array}{c}\text { Reported } \\
\text { (Anjaneyulu et al. 2019) }\end{array}$ & $\begin{array}{c}\text { Reported } \\
\text { (Qiu et al. 2019) }\end{array}$ & $\begin{array}{c}\text { Reported } \\
\text { (Han et al. 2019) }\end{array}$ \\
\hline
\end{tabular}

\begin{tabular}{|c|c|c|c|c|c|c|c|}
\hline Technique & Spectrofluorimetry & HPLC-UV & HPLC-UV & HPLC-UV & HPLC-AER & UPLC-MS/MS & HPLC-FL \\
\hline Application & Dosage form/Urine & Dosage form & Dosage form & Dosage form & Dosage form & Rat plasma & Rat plasma \\
\hline Linearity range & $50-1000 \mathrm{ng} / \mathrm{mL}$ & $100-300 \mu \mathrm{g} / \mathrm{mL}$ & $0.9-5 \mu \mathrm{g} / \mathrm{ml}$ & $3-22 \mu \mathrm{g} / \mathrm{ml}$ & $0.06-2.5 \mu \mathrm{g} / \mathrm{ml}$ & $1-1000 \mathrm{ng} / \mathrm{mL}$ & $2-2000 \mathrm{ng} / \mathrm{mL}$ \\
\hline Organic phase & None & Methanol & Acetonitrile & Acetonitrile & Acetonitrile & Acetonitrile & Acetonitrile \\
\hline
\end{tabular}

Greenness Assessment

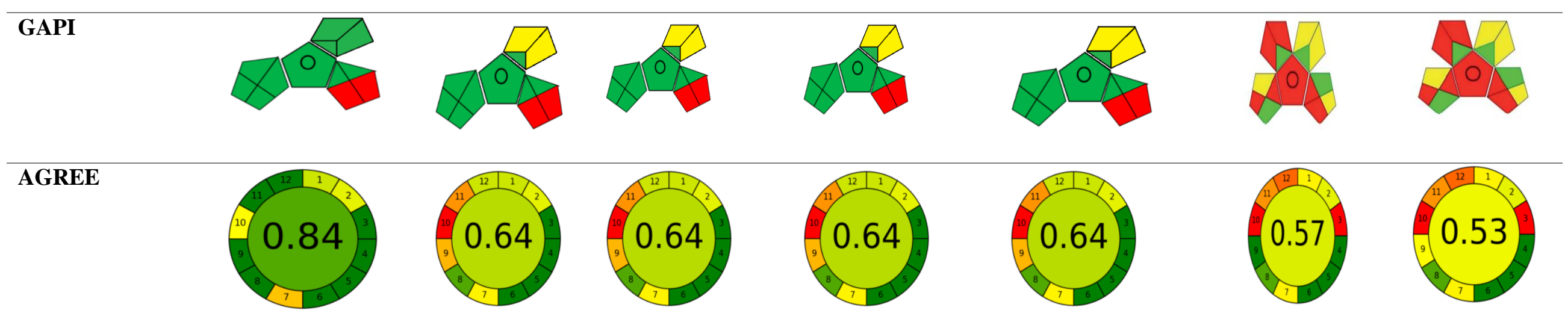


ICH M10 guideline (European Medicines Agency 2019). The Calibration standard samples were prepared by spiking blank urine with appropriate working solutions. Similarly, quality control (QC) samples with four concentration levels were prepared in blank urine at concentrations of 50, 100, 300 , and $500 \mathrm{ng} / \mathrm{mL}$. The calibration curve was constructed with a calibration range of $50-1000$ $\mathrm{ng} / \mathrm{mL}$ then the regression equation was calculated and found to be $y=1.2453 \mathrm{x}+267.52$ where $\mathrm{Y}$ is the relative RFI of ERT and X the concentration of ERT.

The accuracy and precision of the assay were determined via analyzing spiked urine samples at four concentration levels (LLOQ, QCL, QCM, and $\mathrm{QCH})$ using five replicates. Intraday precision was carried out via analysis of the samples on the same day. Whereas inter-day precision was evaluated via repeating analysis over three consecutive days. The regression equation was used to calculate the concentration of ERT in human urine and the summarized data of bioanalytical method parameters are illustrated in Table 2 . The percentage recoveries values were ranged between 99.19-101.90 \% revealing the suitability of the proposed method to quantify ERT in human urine.

\subsection{Application}

\subsubsection{Analysis of pharmaceutical Dosage Form}

The spectrofluorometric method was successfully applied for ERT quantification in a pharmaceutical dosage form. The calculated percentage recoveries and standard deviations were found satisfactory as presented in Table 3. This reveals the accurate quantification of ERT in dosage form by applying the proposed method. Moreover, the proposed method was confirmed by the application of the standard addition technique and the obtained results proved the reliability of the method. The obtained results were found to be acceptable for this method by calculating the percentage recoveries as shown in Table $\mathbf{3}$ revealing no interference from the excipients.

\section{Evaluation of method greenness}

The spectrofluorometric method has been evaluated regarding its greenness using two approaches; green analytical procedure index (GAPI), and analytical greenness metric approach (AGREE) which provide quantitative information on the whole procedure.
The two approaches assess the whole process from transportation, storage, and sample preparation to final determination.

The GAPI pictogram is made primarily of 5 significant pentagrams divided into 15 sections wherein every division addresses an analytical step. GAPI utilizes green, yellow, and red colors to assess the ecological impact, where red addresses bad effects, while yellow and green show medium and low ecological effects, respectively (PłotkaWasylka 2018). As illustrated in Table 4, GAPI evaluation of the spectrofluorometric method shows that 2 red divisions are observed inside the GAPI pictogram which addresses the volume of waste (exceeding 10 milliliters) and treatment of waste since there should be a way for treatment as recycling or passivation.

The rest of the sections show a green color which represents low ecological impact during the specified analytical steps when compared to the other reported methods as represented in Table 4. AGREE diagram composed of an edge of 12 segments; each segment represents one of the green analytical chemistry standards.

The AGREE represents the performance of each analytical step through a color scale (red, yellow, and green) and score (0-1) (Pena-Pereira et al. 2020). As presented in Table 4, the proposed method shows an overall score of 0.84 revealing low ecological impacts compared to other published methods.

\section{Conclusion}

A sensitive, selective, and ecological spectroflourometric method for the detection and quantification of ertugliflozin in dosage form and human urine was developed and fully validated. The simple and fast analytical steps provided a satisfactory recovery. The desired sensitivity of ertugliflozin was achieved with an LLOQ of 50 $\mathrm{ng} / \mathrm{mL}$. The method can be applied for routine bioavailability studies and pharmacokinetics of the cited drug.

\section{Conflict of interest}

The author declares that there is no conflict of interest regarding the publication of this paper. 


\section{References:}

Ahmad I, Mirza T, Anwar Z, Ejaz MA, Sheraz MA, Ahmed S. Multicomponent spectrofluorimetric method for the assay of formylmethylflavin and its hydrolytic products: Kinetic applications. Spectrochim. Acta - Part A Mol. Biomol. Spectrosc. 2018;205:540-50.

Al-Attas AS, Nasr JJ, Shalan S, Belal F. First derivative spectrofluorimetric determination of zopiclone and its degradation product, 2-amino-5chloropyridine, in pharmaceutical formulations with preliminary tool in biological fluids for clinical evidence of zopiclone intake. Spectrochim. Acta Part

A Mol. Biomol. Spectrosc. [Internet]. Elsevier B.V.; 2017;181:148-52. Available from: http://dx.doi.org/10.1016/j.saa.2017.03.036

Anjaneyulu Reddy B., Radhakrishnanand P., Irshad Alam M., Ravi Kiran P. a Validated Reverse-Phase High-Performance Liquid Chromatography-Charged Aerosol Detector Technique for the Simultaneous Estimation of Sitagliptin and Ertugliflozin in Pure and Pharmaceutical Dosage Forms. Asian J. Pharm. Clin. Res. 2019;12(5):236-40.

Cinti F, Moffa S, Impronta F, Cefalo CM, Sun vinsin A, Sorice GP, et al. DDDT-114932-spotlight-onertugliflozin-and-its-potential-in-the-treatmen.

2017;2905-19. Available from:

https://www.ncbi.nlm.nih.gov/pmc/articles/

PMC5633300/pdf/dddt-11-2905.pdf

Dawra VK, Cutler DL, Zhou S, Krishna R, Shi H, Liang Y, et al. Assessment of the Drug Interaction Potential of Ertugliflozin With Sitagliptin, Metformin, Glimepiride, or Simvastatin in Healthy Subjects. Clin. Pharmacol. Drug Dev. 2019a;8(3):314-25.

Dawra VK, Liang Y, Shi H, Bass A, Hickman A, Terra SG, et al. A PK/PD study comparing twicedaily to once-daily dosing regimens of ertugliflozin in healthy subjects. Int. J. Clin. Pharmacol. Ther. 2019b;57(4):207-16.

European Medicines Agency. Biologycal Method Validation M10. Sci. Med. Heal. [Internet]. 2019;44(March). Available from: https://www.ema.europa.eu/en/documents/scientific- guideline/draft-ich-guideline-m10-bioanalyticalmethod-validation-step-2b_en.pdf

European Medicines Agency Committee for Medicinal Products for Human Use (CHMP). European Public Assessment Report (EPAR): Steglatro, INN-ertugliflozin. Procedure No. EMEA/H/C/004315/0000. Eur. Med. Agency [Internet]. 2018;44(January). Available from: http://www.ema.europa.eu/docs/en

GB/document_library/EPAR_Public_assessment_

report/human/004315/WC500246920.pdf

Fediuk DJ, Nucci G, Dawra VK, Cutler DL, Amin NB, Terra SG, et al. Overview of the Clinical Pharmacology of Ertugliflozin, a Novel SodiumGlucose Cotransporter 2 (SGLT2) Inhibitor. Clin. Pharmacokinet. [Internet]. Springer International Publishing; 2020;59(8):949-65. Available from: https://doi.org/10.1007/s40262-020-00875-1

Fediuk DJ, Zhou S, Dawra VK, Sahasrabudhe V, Sweeney K. Population Pharmacokinetic Model for Ertugliflozin in Healthy Subjects and Patients With Type 2 Diabetes Mellitus. Clin. Pharmacol. Drug Dev. 2021;10(7):696-706.

Han DG, Yun H, Yoon IS. A novel highperformance liquid chromatographic method combined with fluorescence detection for determination of ertugliflozin in rat plasma: Assessment of pharmacokinetic drug interaction potential of ertugliflozin with mefenamic acid and ketoconazole. J. Chromatogr. B Anal. Technol. Biomed. Life Sci. [Internet]. Elsevier; 2019;11221123(May):49-57. Available from: https://doi.org/10.1016/j.jchromb.2019.05.023

Harron DWG. Technical Requirements for Registration of Pharmaceuticals for Human Use: The ICH Process. Textb. Pharm. Med. 2013;1994(October 1994):447-60.

Kumari KS, Bandhakavi S. Development and validation of stability-indicating RP-HPLC method for the simultaneous determination of ertugliflozin pidolate and metformin hydrochloride in bulk and tablets. Futur. J. Pharm. Sci. Future Journal of Pharmaceutical Sciences; 2020;6(1):1-10.

Nguyen VK, White JR. Overview of ertugliflozin. Clin. Diabetes. 2019;37(2):176-8. 
P.venkateswara rao, A lakshmana rao S prasad. Simultaneous Estimation of Ertugliflozin and Sitagliptin in Bulk and Pharmaceutical Dosage Form Its. 2018;05(04):2616-27.

Pena-Pereira F, Wojnowski W, Tobiszewski M. AGREE - Analytical GREEnness Metric Approach and Software. Anal. Chem. 2020;92(14):10076-82.

Płotka-Wasylka J. A new tool for the evaluation of the analytical procedure: Green Analytical Procedure Index. Talanta. 2018;181(January):204-9.

Qiu X, Xie S, Ye L, Xu R ai. UPLC-MS/MS method for the quantification of ertugliflozin and sitagliptin in rat plasma. Anal. Biochem. [Internet]. Elsevier Inc.; 2019;567:112-6. Available from: https://doi.org/10.1016/j.ab.2018.12.016

Syed Wajahat S., Aejaz A., G. J. Khan S. and AAQ. Analytical method development and validation for simultaneous estimation of ertugliflozin and metformin $\mathrm{HCl}$ in bulk and pharmaceutical dosage form by HPLC. J. Chem. Inf. Model. 2019;53(9):1689-99.

Zidan DW, Hassan WS, Elmasry MS, Shalaby AA. A novel spectrofluorimetric method for determination of imatinib in pure, pharmaceutical preparation, human plasma, and human urine. Luminescence. 2018;33(1):232-42. 